Revista Brasileira de Agricultura Irrigada v.4, n.2, p.128-138, 2010

ISSN 1982-7679 (On-line)

Fortaleza, CE, INOVAGRI - http://www.inovagri.org.br

Protocolo 030.09 - 28/10/2009 Aprovado em 29/06/2010

\title{
DETERMINAÇÃO DE INTERVALOS ÓTIMOS DE IRRIGAÇÃO UTILIZANDO A BARREIRA LOGARÍTMICA
}

\author{
Angel Ramon Sanchez Delgado ${ }^{1}$, Sérgio Drumond Ventura ${ }^{2}$, Daniel Fonseca de Carvalho ${ }^{3}$ e Ricardo Dias dos \\ Santos ${ }^{4}$
}

\footnotetext{
${ }^{1}$ Professor Associado II, Departamento de Matemática, UFRRJ, Seropédica - RJ, asanchez@ufrrj.br ${ }^{2}$ Professor Adjunto II, Departamento de Matemática, UFRRJ, Seropédica - RJ, ventura@ufrrj.br

${ }^{3}$ Professor Associado II, Departamento de Engenharia, UFRRJ, Seropédica - RJ, carvalho@ufrrj.br. Bolsista do CNPq.

${ }^{4}$ Graduando em matemática na UFRRJ, Bolsista-CNPq CE 203/08, IP 189.74.55.99, rdsantos@ufrrj.br
}

\begin{abstract}
Resumo
Quando a irrigação está restrita pela disponibilidade limitada de água ou pelo limite da capacidade do sistema de irrigação e a terra é o fator limitante da produção, a água economizada pela redução da profundidade de irrigação pode ser utilizada para irrigar um acréscimo de terra. Nesse caso, o problema é determinar uma lâmina ótima em relação à profundidade aplicada e a área a ser irrigada; isto é, procura-se maximizar a receita líquida total sujeita às restrições de recursos pré-fixados e a uma estrutura de preços e custos prédeterminados. Neste trabalho, apresentamos um procedimento computacional baseado no método barreira logarítmica (MBL) para a procura de intervalos ótimos de irrigação entre a lâmina que maximiza a produção e a lâmina que maximiza a receita líquida com limitações hídricas. Foram realizados três experimentos numéricos baseados nas funções de resposta quadráticas das culturas: algodão, trigo e melancia, para esta última foi considerada uma variação de preços e custos de água. Considerando que os intervalos ótimos de irrigação obtidos são completamente compatíveis com os apresentados na literatura, pode-se concluir que o procedimento computacional e iterativo tipo barreira logarítmica implementado na linguagem octave, constitui uma alternativa confiável na tomada de decisões econômicas que se apresentam em um planejamento ótimo de irrigação com déficit.

Palavras-Chave: Irrigação otimizada, lâmina de irrigação, pontos interiores.
\end{abstract}

\section{ABSTRACT \\ DETERMINATION OF GREAT INTERVALS OF IRRIGATION USING THE LOGARITHMIC BARRIER}

When the irrigation is restricted for the limited readiness of water or for the limit of the capacity of the overhead irrigation, the water saved by the reduction of the irrigation depth can be used to irrigate an earth increment. In that I annul, the problem is to determine a great sheet in relation to the applied depth and the area to be irrigated; that is, tries to maximize the 


\section{DETERMINAÇÃO DE INTERVALOS ÓTIMOS DE IRRIGAÇÃO UTILIZANDO A BARREIRA LOGARÍTMICA}

income liquid total subjects to the restrictions of pre-fastened resources and the a structure of prices and predetermined costs. In this work, we presented a procedure computational based on the method logarithmic barrier (MBL) for the search of great intervals of irrigation among the sheet that maximizes the production and the sheet that it maximizes the liquid income with limitations hidrics. Three numeric experiments were accomplished based in the quadratic answer functions of the cultures: wheat, cotton and watermelon, for this last one were considered a variation of prices and costs of water. Considering that the great intervals of irrigation obtained healthy completely compatible with introduced them in the literature, it can be concluded that the procedure computational and iteration type logarithmic barrier implemented in the language octave, it constitutes a reliable alternative in the socket of economical decisions that you/they come in a great planning of irrigation with deficit.

Key words: Optimized irrigation, irrigation sheet, interior points.

\section{INTRODUÇÃO}

Em regiões áridas ou semi-áridas e também em regiões úmidas, a irrigação com déficit depende da utilização racional dos recursos. A maioria das culturas possui períodos críticos, durante as quais o suprimento inadequado de água causa reduções na produção e alterações no desenvolvimento das culturas. Segundo ENGLISH M. \& RAJA (1996) lâminas excessiva de água além de carregar mais custos na produção, também são prejudiciais por reduzirem o rendimento da cultura; por outro lado, lâminas insuficientes expõe a cultura a condições de deficiência hídrica, reduzindo seu potencial produtivo.

Considerando que o comportamento de uma cultura depende da quantidade e frequência de irrigação que sejam administrados durante o ciclo fenológico; as funções de produção, de receitas e custos representam ferramentas fundamentais nos estudos econômicos relativos ao planejamento da irrigação. Se estas funções fossem conhecidas com precisão, seria possível selecionar com exatidão o nível ótimo de água para uma situação em particular; mas, tais funções estão restritas a grandes variações, dificultando as previsões. Variações climáticas, atributos físicos e hídricos do solo, uniformidade de distribuição da água pelo sistema de irrigação e muitos outros fatores, fazem difícil prever a produtividade das culturas. Da mesma maneira, por serem variáveis os preços e os insumos agrícolas, um elevado grau de incerteza é obtido nas estimativas (FRIZZONE et al. 2005). A incerteza na produção está associada ao risco econômico e através de estratégias da irrigação com déficit é possível sua redução, mas não sua eliminação.

Segundo FRIZZONE et al. (2005) este é um problema relevante em irrigação, pois não se pode conhecer com exatidão a forma da curva de produção em função da água aplicada que maximizará a receita líquida. Também, a quantidade ótima de água representa apenas um ponto sobre a curva de produção, tendo pouco significado prático. Mesmo assim, é possível determinar um intervalo no qual a receita líquida é maior que aquela obtida quando se utiliza a quantidade de água que maximiza a produção $\left(w_{m}\right)$.

Nos últimos quarenta anos a literatura, bem como (ENGLISH, 1990), vem mostrado que em certas circunstâncias, as economias do déficit de irrigação se encontram na eficiência da irrigação aumentada, nos custos reduzidos de irrigação e nos custos oportunos da água. Adicionalmente, uma decisão que usa menos água pode permitir ao produtor reduzir o capital e outros custos fixos. A experiência prática diz que quando a terra constitui um fator limitante à produção, a utilização de irrigação com déficit permite um maior retorno econômico que a 


\section{DETERMINAÇÃO DE INTERVALOS ÓTIMOS DE IRRIGAÇÃO UTILIZANDO A BARREIRA LOGARÍTMICA}

irrigação completa (ENGLISH et al. 1990, 1996, 2002). As vantagens potenciais do déficit de irrigação parecem bastante significantes, particularmente em uma situação onde a água e/ou a terra é limitada e os riscos associados são aceitáveis.

Supondo que o sistema de irrigação utilizado aplica água segundo um modelo teórico de distribuição, uma lâmina de água ótima deve ser infiltrada, de forma a minimizar a redução da receita líquida esperada, quando temos um déficit de água na área. Esta lâmina é chamada de lâmina ótima de irrigação, ou seja, é a lâmina média de água que deve ser infiltrada no solo para proporcionar a máxima receita líquida, de maneira a minimizar a redução da receita líquida pelo déficit de água.

Se a terra é limitada, a estratégia de irrigação ótima é a quantia de água máxima para a receita líquida derivada de cada unidade de terra; isto é, o máximo diferencial entre as curvas descritas pela forma quadrática da função receita e a forma linear da função de custos. Tal quantia é denotada por $w_{*}$ e é pouco menos que $w_{m}$, considerando que ambas as curvas decrescem à esquerda de $w_{m}$ (ENGLISH, 1990). Se usarmos mais água que $w_{m}$ o lucro é reduzido. De acordo com a teoria econômica, $w$, é a lâmina onde o valor do produto marginal é igual ao valor do custo marginal; geometricamente, quer dizer onde o declive da função custo é igual ao declive da curva receita líquida.

Se o uso da água é reduzido abaixo de $w_{*}$, uma lâmina de água pode ser encontrada de maneira que a receita líquida por unidade de terra seja igual à receita líquida em $w_{m}$. Esta lâmina será denotada por $w_{\boldsymbol{e}}$ * e é chamada de equivalente. No intervalo $\left[w_{e *}, w_{m}\right]$ a irrigação parcial (com déficit) será mais rentável que a irrigação plena (para a máxima produção). (ENGLISH, 1990)

A extensão desse intervalo pode ser interpretada como uma indicação do grau de segurança no manejo da irrigação parcial. Se o intervalo é relativamente amplo, o risco associado à decisão pode ser pequeno. Um intervalo pequeno implica maior risco e necessidade de um cuidadoso manejo da irrigação. Dessa forma, conhecido esse intervalo e a incerteza da estimativa da quantidade ótima de água, a decisão de quanto irrigar pode ser tomada com mais segurança (FRIZZONE et al. 2005).

O MBL é o mais antigo método de pontos interiores, com prestígio radicalmente incrementado após a revolução de KARMARKAR (1984). MBL é um processo de penalização. Nele, a não satisfação (ou o "risco de não-satisfação") de uma restrição é sancionada com um acréscimo da função objetivo, de maneira que a função que define a restrição é eliminada como tal e substituída por um termo introduzido no objetivo, que tende a menos infinito quando o ponto se aproxima da fronteira do conjunto factível. As estratégias de penalização estão vivas na otimização contemporânea por sua simplicidade e capacidade de se enriquecer automaticamente com progressos realizados na resolução de problemas mais simples.

Com isso, um procedimento computacional decorrente do método barreira logarítmica (MBL) é apresentado neste trabalho com o objetivo de determinar intervalos ótimos de irrigação baseados no cálculo da lâmina de água que maximiza a produção e a lâmina de água que maximiza a receita líquida com restrições hídricas quando a disponibilidade de terra é fator limitante da produção e existe uma estrutura de custos e preços pré-fixados.

\section{MATERIAL E MÉTODOS}

Denotemos com $\mathrm{y}(w)$ a função de produção ou resposta de uma determinada cultura $\left(\mathrm{kg} \cdot \mathrm{ha}^{-1}\right)$ em relação à lâmina de água $w(\mathrm{~mm}$ ou $\mathrm{cm})$; em geral, uma função não linear e $w_{l}, w_{u}$ limitantes inferiores e superiores de $w$, sendo $w_{l} \geq 0$ e $w_{u}>$ $w_{l}$. 


\section{DETERMINAÇÃO DE INTERVALOS ÓTIMOS DE IRRIGAÇÃO UTILIZANDO A BARREIRA LOGARÍTMICA}

Seja $w_{m}=\operatorname{Argmax}\left\{\mathrm{y}(w): w_{l} \leq w\right.$ $\left.\leq w_{u}\right\}$ e $y_{m}=\mathrm{y}\left(w_{m}\right)$. Aqui Argmax $\{$. denota a variável ou argumento; neste caso lâmina de água, onde se alcança o valor objetivo máximo (produção) satisfazendo as limitações impostas. Neste trabalho é considerado receita líquida $\left(\mathrm{US} \$ . \mathrm{ha}^{-1}\right.$ ou $\left.\mathrm{US} \$ \cdot \mathrm{m}^{-3}\right)$ à relação dada por: $\mathrm{I}(w)=\frac{w_{T}}{w}\left(\mathrm{P}_{\mathrm{c}}\right.$ $\left.\mathrm{y}(w)-c_{0}-c_{w^{*}} w\right)$ em que $w_{T}\left(\mathrm{~m}^{3}\right)$ representa o total de água disponível, $\mathrm{P}_{\mathrm{c}} \mathrm{O}$ preço da cultura (US $\left.\$ . \mathrm{kg}^{-1}\right), c_{0}$ o custo fixo de produção (US\$.ha ${ }^{-1}$ ) e $c_{w}$ o custo de água US\$.(mm.ha) ${ }^{-1}$. É conhecido na literatura que a lâmina de água que maximiza a receita líquida representa uma lâmina ótima na irrigação com déficit; isto é, interessa calcular aproximadamente $w_{*}$. $=\operatorname{Argmax}\left\{\mathrm{I}(w): w_{l} \leq \mathrm{w} \leq w_{u}\right\}$ e $I_{*}=$ $\mathrm{I}\left(w_{*}\right)$ quando a disponibilidade de terra é o fator limitante à produção. Outra lâmina de interesse é aquela que produz receita líquida $\mathrm{I}(w)$, igual a $\mathrm{I}\left(w_{m}\right)$. Esta lâmina equivalente foi denotada por $w_{e *}$. Serão consideradas funções de produção na forma quadrática; $\mathrm{y}(\mathrm{w})=\mathrm{a} w^{2}+\mathrm{b} w+\mathrm{c}$. Diversos trabalhos mostram que para a função produção água cultura, um modelo polinomial de segundo grau é normalmente utilizado.A continuação desenvolve-se um procedimento computacional iterativo que determina as lâminas de água $w_{m}, w_{*} \mathrm{e}$ $w_{e *}$, assim como os respectivos valores da produtividade e da receita líquida maximizada quando a disponibilidade de terra é fator limitante. $\mathrm{O}$ procedimento tem por base a metodologia barreira logarítmica que funciona da seguinte maneira: Fixando um parâmetro $\mu>0$ e incorporando as restrições hídricas na função objetivo através de um função "barreira logarítmica", se obtém $w(\mu)=\operatorname{Argmax}\left\{\Phi_{\mu}(w): w_{l}<w<w_{u}\right.$ \} em que $\Phi_{\mu}=\mathrm{y}(w)+\mu . \mathrm{B}(w)$ para o caso da maximização da produção e $\Phi_{\mu}=\mathrm{I}(w)+$ $\mu . \mathrm{B}(w)$ para o caso da receita líquida máxima. Em ambos os casos, $\mathrm{B}(w)=\log (w$ $\left.-w_{l}\right)+\log \left(w_{u}-w\right)$. Em seguida faz-se um decréscimo de $\mu$ e o processo é repetido até que um critério de parada prédeterminado seja satisfeito. $O$ nome barreira logarítmica deve-se ao fato que a função logaritmo obriga ao procedimento a gerar pontos interiores do intervalo [ $w_{l}$, $w_{u}$ ]. Para cada $\mu$ o máximo de $\Phi_{\mu}$ é alcançado em um ponto interior e quando $\mu$ tende a zero, este ponto move-se até um ponto próximo da solução ótima do problema. $\mathrm{E}$ ainda, o conjunto $\mathrm{T}_{\mathrm{c}}=\{w(\mu)$ $/ \mu>0\}$ define uma curva suave denominada trajetória central associada à produção ou à receita líquida respectivamente. (GONZAGA C. C. 1992) No caso da maximização da produção, $w=$ $w(\mu)$ define uma solução ótima se $\mathrm{e}$ somente se:

$$
w-\frac{1}{2 a}\left(\boldsymbol{\mu} \frac{\bar{E}-w_{l}}{w}-\boldsymbol{\mu} \frac{\overline{\underline{B}}}{w_{u}-w}\right)=\frac{-b}{2 a} .
$$

Fazendo $s_{l}=\boldsymbol{\mu} \frac{\overline{\underline{B}}}{w-w_{l}}$ e $s_{u}=\boldsymbol{\mu} \frac{\overline{\bar{B}}}{w_{u}-w}$ temos que as condições necessárias e suficientes são:

$$
\begin{aligned}
& \frac{1}{2 a}\left(s_{l}-s_{u}\right)=\frac{-b}{2 a} \\
& s_{l}\left(w-w_{l}\right)=\mu \\
& s_{u}\left(w_{u}-w\right)=\mu
\end{aligned}
$$

Os pontos que resolvem (1) - (2) - (3) encontram-se próximos da trajetória central $\mathrm{T}_{\mathrm{c}}$ associada à produtividade; mais ainda, a (1) representa a restrição que define a região de viabilidade do correspondente problema dual; enquanto (2) - (3) representam as condições de "folgas complementares aproximadas". Entre as vantagens das soluções duais destacam-se as possibilidades de fornecer informações econômicas sobre a utilização de recursos; como por exemplo, auxiliar na tomada de decisão para a aquisição de recursos adicionais ou na análise de sensitividade. De (1) se tem que $\frac{1}{2 a}\left(s_{u}-s_{l}\right)$ representa a diferença entre a lâmina onde se alcança a máxima produção 


\section{DETERMINAÇÃO DE INTERVALOS ÓTIMOS DE IRRIGAÇÃO UTILIZANDO A BARREIRA LOGARÍTMICA}

sem restrições e a lâmina obtida na iteração atual.

Para o caso da maximização da receita líquida, $w=w(\mu)$ define uma solução ótima se e somente se:

$w^{2}\left(s_{l}-s_{u}-p_{c} a\right)=w_{T}\left(p_{c} c-c_{0}\right)$

Pode-se dizer que $o$ procedimento numérico implantado para a determinação das lâminas $w_{m}, w_{*} e w_{e}$. funciona como um método de duas fases. Em uma primeira fase resolvemos iterativamente $o$ sistema não linear (1) - (2) - (3) usando o método de Newton e decrescendo o valor de $\mu>\mathbf{0}$ até satisfazer certo critério de parada. Desta maneira se obtém o valor da lâmina $w_{m}$. Seguidamente em uma segunda fase faz-se $w_{T}=w_{m}$ e resolve iterativamente o sistema (2) - (3) - (4), também com decréscimo do parâmetro $\mu$, para obter o valor da lâmina $w_{*}$. Finalmente resolvendo a equação de segundo grau $\mathrm{I}(w)=\mathrm{I}\left(w_{m}\right)$, é determinado o valor da lâmina equivalente $w_{e *}$. Mais detalhes no Apêndice.

Para pesquisar o desempenho do procedimento implementado, foram selecionados os dados apresentados em ENGLISH M. \& RAJA (1996) pra as culturas do trigo e do algodão, como também os apresentados em ANDRADE JÚNIOR et al. (2001) para a melancia. Conforme mostra a Tabela 1.

Tabela 1. Funções de resposta das culturas em relação à lâmina de água aplicada

\begin{tabular}{|c|c|c|c|}
\hline Culturas & Equações & $\begin{array}{l}P_{c} \\
\left(U S \$ . \mathrm{kg}^{-1}\right)\end{array}$ & $\begin{array}{l}c_{0} \\
\left(U S \$ . h a^{-1}\right)\end{array}$ \\
\hline Algodão & $y(w)=-0,091 w^{2}+29,85 w-781,1(1)$ & 1,59 & 770 \\
\hline Trigo & $y(w)=-0,00273 w^{2}+0,3326 w-0,5348$ & 147 & 482,30 \\
\hline Melancia & $y(w)=-0,32089 w^{2}+228,624 w+3,137$ & - & - \\
\hline
\end{tabular}

O preço adotado para o algodão foi de 1,59 US\$. $\mathrm{kg}^{-1}$. O custo fixo foi de 770 US\$ e o custo de água de 7,30 US\$.(cm.ha) $)^{-1}$, considerando um intervalo de irrigação de [ 42.2, 70 ]. Para o trigo foi considerado

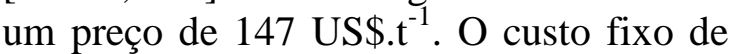
produção foi de 482,30 US\$ e o custo de água de 7,79 US\$.(cm.ha) $)^{-1}$, com um intervalo de irrigação de [ 100 , 300 ]. Já para a melancia foram considerados preços entre 0,05 US\$. $\mathrm{kg}^{-1}$ e $0,35 \mathrm{US} \$ . \mathrm{kg}^{-1}$ e três custos de água quando consideram-se três tarifas de energia elétrica: 0,2748
US\$.(mm.ha) $)^{-1} ; 0,1546$ US\$.(mm.ha) $)^{-1}$ e $0,1448$ US\$.(mm.ha) $)^{-1}$ e um intervalo de irrigação de $[0,700]$.

\section{RESULTADOS E DISCUSSÃO}

Foram analisados três cenários hidroagrícolas diferentes, onde a programação foi realizada na linguagem computacional octave. Os resultados quanto a produtividade, receitas líquidas e suas respectivas lâminas de água são apresentados na Tabela 2.

Tabela 2. Valores referentes à produtividade, às receitas líquidas e suas respectivas lâminas de água.

\begin{tabular}{|c|c|c|c|c|c|c|}
\hline Cultura & $\begin{array}{l}y(w) \\
\text { kg.ha }^{-1}\end{array}$ & $I_{m}(w)$ \$.ha & $\begin{array}{l}\text { I. }(w) \\
\$ . h^{-1}\end{array}$ & $\begin{array}{l}\boldsymbol{w}_{\boldsymbol{m}} \\
\mathrm{cm} \cdot \mathrm{ha}^{-1}\end{array}$ & $\begin{array}{l}\text { w. } \\
\mathrm{cm} \cdot \mathrm{ha}^{-1}\end{array}$ & $\begin{array}{l}\boldsymbol{w}_{e} \\
\mathrm{~cm} \cdot \mathrm{ha}^{-1}\end{array}$ \\
\hline Algodão & $1.666,8$ & 682,88 & 774,95 & 164,011 & 138,7847 & 113,5583 \\
\hline Trigo & $9.595,5$ & 453,73 & 491,51 & 60,913 & 51.21 & 41,5 \\
\hline Melancia & 43.855 & - & - & 356,2186 & - & - \\
\hline
\end{tabular}




\section{DETERMINAÇÃO DE INTERVALOS ÓTIMOS DE IRRIGAÇÃO UTILIZANDO A BARREIRA LOGARÍTMICA}

Para a cultura do algodão o valor da produtividade máxima após a implementação do procedimento MBL em foi de $1.666,8 \mathrm{~kg} \cdot \mathrm{ha}^{-1}$, com a lâmina de $164,011 \mathrm{~cm} \cdot \mathrm{ha}^{-1}$, produzindo uma receita líquida de 682,88 US\$.(cm.ha) ${ }^{-1}$. O mesmo procedimento permitiu obter uma receita líquida máxima de 774,95 US\$.(cm.ha) $)^{-1}$ com uma lâmina ótima de $138,7847 \mathrm{~cm} \cdot \mathrm{ha}^{-}$ 1. Também a lâmina equivalente foi de $113,5583 \mathrm{~cm} \cdot \mathrm{ha}^{-1}$. Os resultados são compatíveis com os obtidos em ENGLISH \& RAJA (1996), onde para a lâmina máxima de $164 \mathrm{~cm} \cdot$ ha $^{-1}$ obtém uma receita líquida de 682,87 US\$.(cm.ha $)^{-1}$,e para uma lâmina ótima de 139 mm.ha ${ }^{-1}$ uma receita máxima de 774,96 US\$.(cm.ha) ${ }^{-1}$. A lâmina equivalente ficou em $114 \mathrm{~cm} \cdot \mathrm{ha}^{-1}$. Obtendo assim um intervalo ótimo de irrigação [164,011 ; 113,5583], onde se obtém uma maior rentabilidade que a irrigação máxima, que é compreendida entre a lâmina equivalente e a lâmina máxima.

A vantagem de utilizar uma metodologia que segue iterativamente a trajetória central é que ela permite visualizar a convergência à solução quando $\mu$ decresce até uma tolerância predeterminada. Mais ainda, a cada iteração é dada ciência do processo das soluções aproximadas geradas, e da região onde se encontram valores ótimos dos problemas de produção e receita líquida.

$\mathrm{Na}$ Figura 1 pode-se observar um pequeno esboço da trajetória central gerada $(w(\mu), \mathrm{y}(w(\mu)))$ para cada $\mu$ fixo, neste caso da função produtividade $\mathrm{y}(w)$. $\mathrm{Na}$ figura 2 mostra-se a trajetória central para a receita líquida $(w(\mu), I(w(\mu)))$ para cada $\mu$ fixo. Da mesma maneira para a cultura do trigo foi obtida a produtividade máxima de 9.5955 t.ha $^{-1}$ com a lâmina de 60,913 $\mathrm{cm} \cdot \mathrm{ha}^{-1}$, e uma receita líquida de 453,73 US\$.(cm.ha) $)^{-1}$. A metodologia permitiu obter uma lâmina ótima de $51.21 \mathrm{~cm} \cdot \mathrm{ha}^{-1} \mathrm{e}$ uma receita líquida máxima de 491,51 US\$.(cm.ha) ${ }^{-1}$. A lâmina equivalente ficou em $41,5 \mathrm{~cm} \cdot \mathrm{ha}^{-1}$. Com um intervalo ótimo de irrigação $[60,913 ; 41,5]$. Novamente os resultados são compatíveis com os obtidos em ENGLISH \& RAJA (1996), onde para a lâmina máxima de $61 \mathrm{~cm} \cdot \mathrm{ha}^{-1}$, obtém-se uma receita líquida de 453,70 US\$.(cm.ha) ${ }^{-1}$. A receita líquida máxima de 491,51 US\$.(cm.ha) ${ }^{-1}$ é obtida com a lâmina ótima de $51 \mathrm{~cm} \cdot \mathrm{ha}^{-1}$, e a lâmina equivalente igual a $42 \mathrm{~cm} \cdot \mathrm{ha}^{-1}$.

De modo análogo às figuras 1 e 2 , nas figuras 3 e 4 mostra-se a trajetória central gerada pela resposta das funções produtividade $\mathrm{y}(w)$ e receita líquida $\mathrm{I}(w)$ respectivamente.

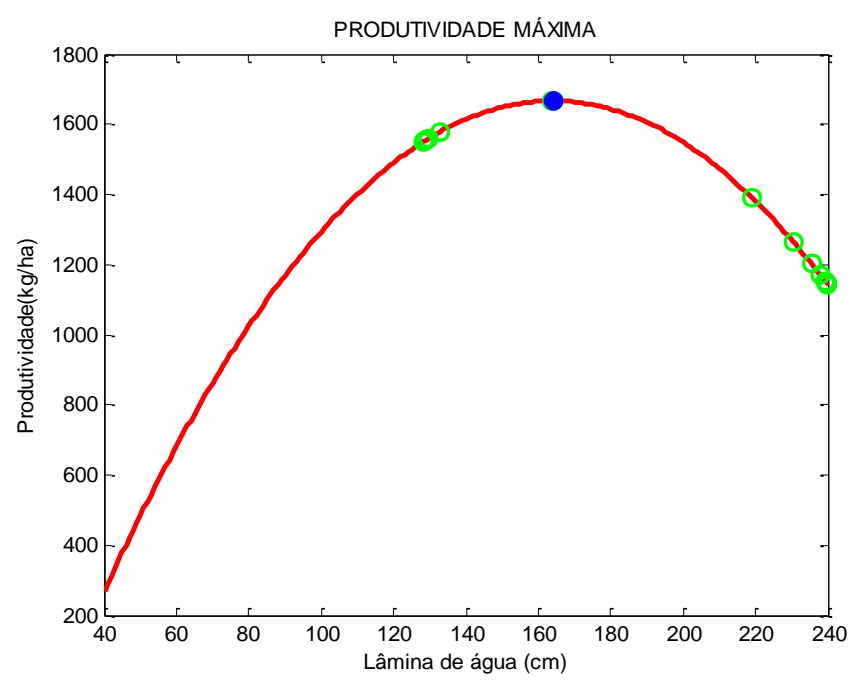

Figura 1 - Curva de resposta da função de produtividade y $(w)$ e a trajetória central associada à produtividade do algodão. 


\section{DETERMINAÇÃO DE INTERVALOS ÓTIMOS DE IRRIGAÇÃO UTILIZANDO A \\ BARREIRA LOGARÍTMICA}

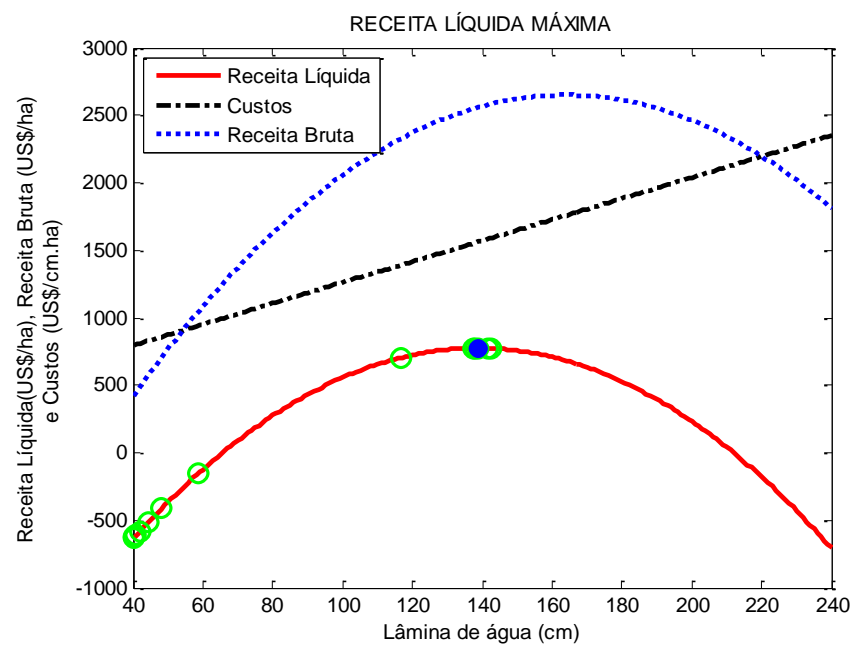

Figura 2 - Curva de resposta da função receita líquida $\mathrm{I}(w)$ e a trajetória central associada à receita líquida do algodão.

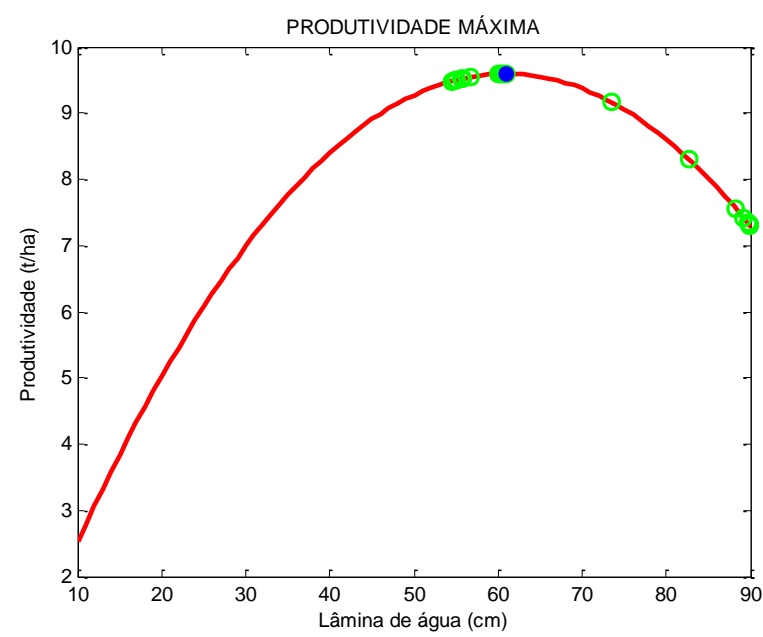

Figura 3 - Curva de resposta da função de produtividade $\mathrm{y}(w)$ e a trajetória central associada à produtividade do trigo.

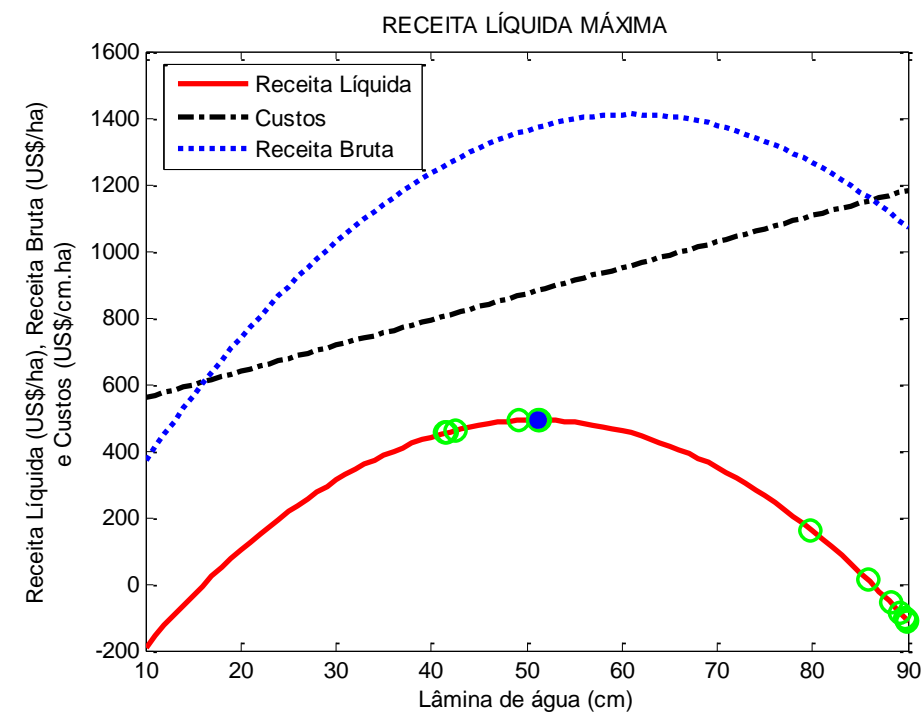

Figura 4 - Curva de resposta da função receita líquida $\mathrm{I}(w)$ e a trajetória central associada à receita líquida do trigo. 


\section{DETERMINAÇÃO DE INTERVALOS ÓTIMOS DE IRRIGAÇÃO UTILIZANDO A BARREIRA LOGARÍTMICA}

Para a cultura da melancia obteve-se uma lâmina máxima de água igual a 356, $2186 \mathrm{~mm}$ com produção de 43.855 $\mathrm{kg} \cdot \mathrm{ha}^{-1}$. Essa lâmina de irrigação situa-se dentro da faixa considerada ideal para a cultura $(300 \quad \mathrm{~mm}$ a $400 \mathrm{~mm})$ (DOOREMBOS \& KASSAM, 1994).

Como é mostrado em ANDRADE JÚNIOR et al. (2001), a lâmina ótima para manejo da irrigação depende do preço da cultura e a medida que o preço aumenta as lâminas $w_{*}$ e $w_{e}$ * diminuem, aumentando o intervalo de manejo racional da água. Desta maneira, a economia da água com a aplicação de $w_{*}$ e $w_{e *}$ varia de acordo ao preço da cultura. Também mostram que para preços maiores ou iguais que 0,40
US\$.kg ${ }^{-1}$, os valores de $w_{e}$ * aproximam-se de zero, inviabilizando a adoção do manejo econômico da irrigação. Nesse caso, a estratégia ótima de irrigação consiste na aplicação da lâmina $w_{m}$ uma vez que o cultivo de melancia torna-se uma atividade de alto valor econômico (YARON \& BRESLER 1983).

Os valores das lâminas máxima, ótima e equivalente, correspondentes às estratégias de irrigação, e as respectivas receitas líquidas obtidas em razão das diferentes combinações de preço e custo da água nas três formas de tarifação da energia elétrica (normal, verde no horário de pico e verde fora da hora de pico) são apresentadas na Tabela 1.

Tabela 1. Resultados das estratégias de irrigação $(w)$ e respectivas receitas líquidas (US $\$ . \mathrm{m}^{-3}$ ) com as diferentes combinações de preço da cultura $\mathrm{P}_{\mathrm{c}}$ e o custo da água $\left(c_{W}\right)$ nas três formas de tarifação da energia elétrica.

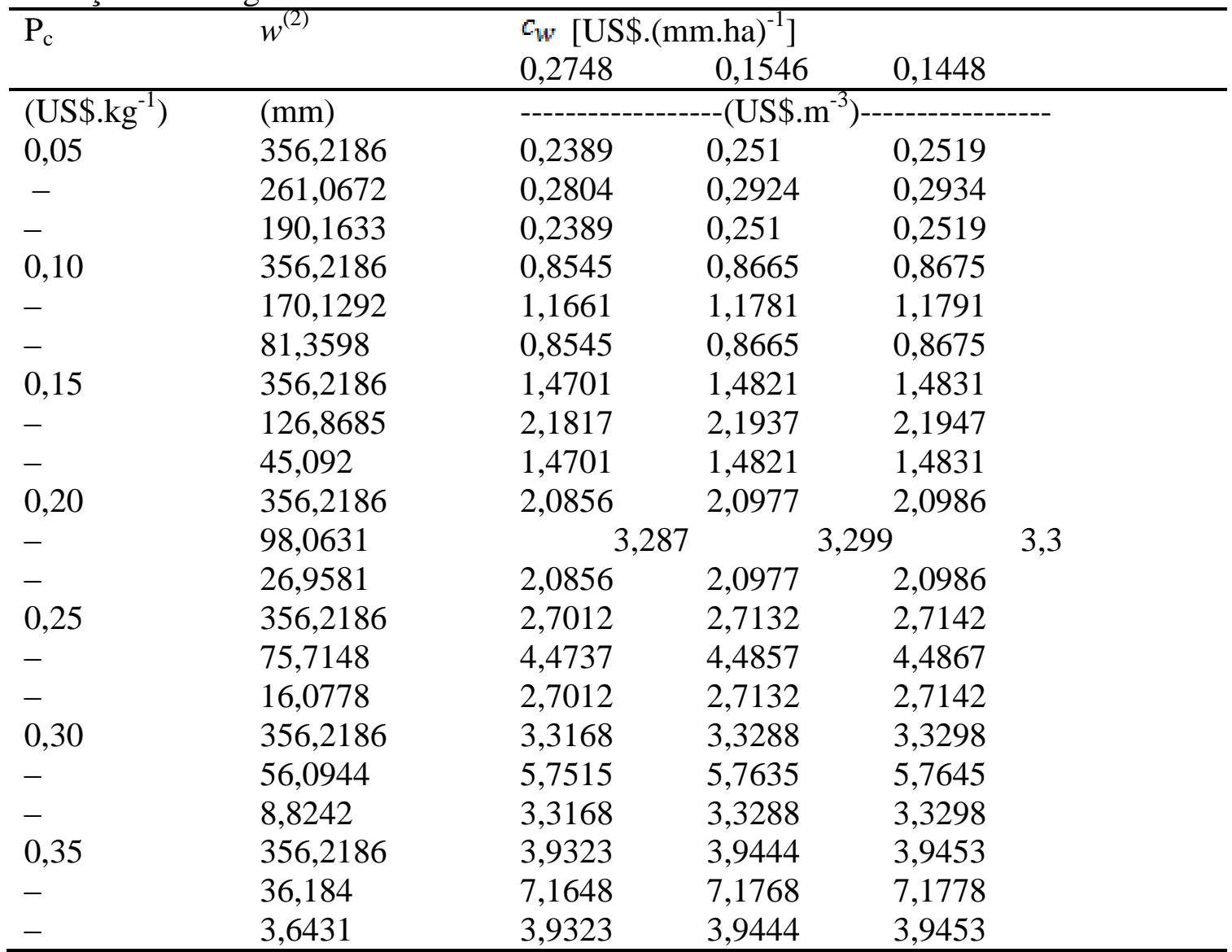

Em cada valor $\mathrm{P}_{\mathrm{c}}$, os valores de $w$ Os intervalos de manejo racional de água correspondem às lâminas máxima, ótima e variam de $356,2 \mathrm{~mm}$ a $3,6431 \mathrm{~mm}$ em equivalente. 


\section{DETERMINAÇÃO DE INTERVALOS ÓTIMOS DE IRRIGAÇÃO UTILIZANDO A BARREIRA LOGARÍTMICA}

razão da oscilação do preço da cultura. Os limites inferiores de cada intervalo representam a lâmina equivalente, enquanto os limites superiores

correspondem à lâmina que maximiza a produção.

Se considerarmos como em ANDRADE JÚNIOR et al. (2001) que o preço médio da melancia é de 0.15 US\$. $\mathrm{kg}^{-1}$, o intervalo de manejo racional de água varia entre 45,092 $\mathrm{mm}\left(w_{e *}\right)$ e $356,2186 \mathrm{~mm}$. Nesse caso a lâmina ótima é de $126,8685 \mathrm{~mm}$ e a receita líquida de 2,1817 US\$.m ${ }^{-3}$. Desta maneira o produtor poderá adotar a irrigação com déficit e aumentar a área irrigada com o volume de água que foi economizado. Como é indicado em ANDRADE JÚNIOR et al. (2001), essa estratégia é viável na faixa de variação de preços de $0,05 \mathrm{US} \$ . \mathrm{kg}^{-1}$ a $0,35 \mathrm{US} \$ \cdot \mathrm{kg}^{-1}$. Igual que o reportado em ANDRADE JÚNIOR et al. (2001), a Tabela 1 nos mostra que para o preço baixo de 0,05 US\$. $\mathrm{kg}^{-1}$ a receita líquida obtida com a lâmina ótima $\left(0,2804\right.$ US $\left.\$ . \mathrm{m}^{-3}\right)$ foi superior em mais de $16 \%$ que a receita líquida proporcionada com a aplicação da lâmina que maximiza a produção $\left(0,2389\right.$ US\$.m ${ }^{-}$ $\left.{ }^{3}\right)$. Também, para o preço elevado de 0,35 US\$. $\mathrm{kg}^{-1}$ a receita líquida obtida com a lâmina ótima $\left(7,16\right.$ US\$. $\left.\mathrm{m}^{-3}\right)$ foi superior em mais de $80 \%$ que a receita líquida proporcionada com a aplicação da lâmina que maximiza a produção $\left(3,9323\right.$ US\$.m ${ }^{-}$ $\left.{ }^{3}\right)$. Isto nos indica que dentro do intervalo de preços definido, à medida que o preço aumenta, torna-se mais recomendável optar pela irrigação com déficit.

$\mathrm{Na}$ figura 5 observa-se o esboço da trajetória central de pontos gerada $(w(\mu)$, $\mathrm{y}(w(\mu)))$ para cada $\mu$ fixo que converge à lâmina máxima, neste caso a máxima produtividade fisiológica da melancia.

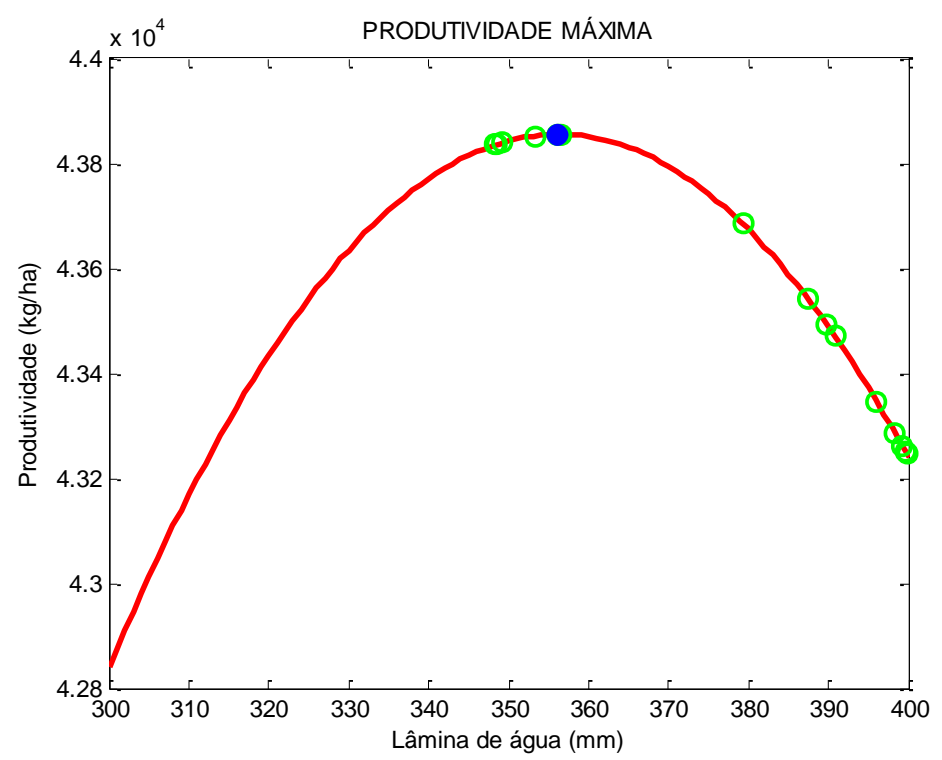

Figura 5 - Curva de resposta da função de produtividade $\mathrm{y}(w)$ e a trajetória central associada à produtividade da melancia.

\section{CONCLUSÃO}

Considerando que os resultados obtidos para esta experiência numérica são inteiramente compatíveis com os apresentados na literatura citada e que a implementação metodológica para a determinação da lâmina de água que maximiza a produtividade e a receita líquida com restrições hídricas é simples (ver Apêndice), pode-se concluir que o procedimento computacional iterativo utilizando o método da Barreira Logarítmica (MBL) aqui apresentado constitui uma alternativa confiável, com 


\section{DETERMINAÇÃO DE INTERVALOS ÓTIMOS DE IRRIGAÇÃO UTILIZANDO A BARREIRA LOGARÍTMICA}

boa interface de implementação na determinação de intervalos ótimos de irrigação. Sendo satisfatório na obtenção dos intervalos ótimos de irrigação com uma rentabilidade acima da obtida com a irrigação máxima.

\section{APÊNDICE}

$\mathrm{O}$ procedimento iterativo implementado na linguagem octave escolhe inicialmente os valores dos parâmetros que definem a função resposta da cultura considerada ( $a, b, c, \in \mathbf{R}$ ), os limitantes inferiores e superiores da lâmina de água $\left(w_{l}, w_{u} \in \mathbf{R}_{+}\right)$, o parâmetro que define a trajetória central $(\mu>0)$, um ponto inicial dentro do intervalo de restrições $\left(w_{0}\right)$ e dois parâmetros suficientemente pequenos associados à redução do parâmetro de penalidade logarítmica $\left({ }^{\delta}\right)$ e à condição de parada $(\varepsilon)$. Em seguida verifica-se a condição de parada, isto é, se as brechas associadas à viabilidade dual $(\rho, \sigma)$ e folgas complementares $\left(f_{\mu}^{1}, f_{\mu}^{2}, f_{\mu}^{3}, f_{\mu}^{4}\right)$ têm sido reduzidas suficientemente. Caso contrário, calcula-se uma direção Newton e um tamanho de passo. Atualizam-se os pontos soluções, acha-se uma redução do parâmetro de penalidade e finalmente calcula-se novamente a condição de parada.

\section{PROCEDIMENTO}

Dados a,b,c $\in \mathbf{R} ; \mathrm{a}, \mathrm{c}<0 ; \mathrm{b}>0 ; \mathrm{P}_{\mathrm{c}} ; c_{w}, c_{0} \in \mathbb{R}_{+} ; \mu_{0}>0, w_{l}, w_{u} \in \mathbb{R}_{+} ; w_{l}<w_{u} ;$ $\varepsilon, \delta \in(0,1) ; w_{0}=\frac{w_{u}+w_{l}}{2}$

FASE 1 ( Cálculo da lâmina que Maximiza a Produção da Cultura em [ $\left.w_{l}, w_{u}\right]$ )

$$
\begin{array}{rlrl} 
& \text { Fazer } & w & =w_{0}, s_{l}=s_{l}^{0}, s_{u}=s_{u}^{0} \mu=\mu_{0} \\
& \text { Calcular } \quad \rho & =-b-2 a w-s_{l}+\frac{s_{u}}{2 a} \\
& f_{\mu}^{1} & =\mu-\left(w-w_{l}\right) s_{l} ; f_{\mu}^{2}=\mu-\left(w_{u}-w\right) s_{u} ;
\end{array}
$$

Calcular

$$
\frac{\frac{s_{l}^{0}=\mu_{0}}{w_{0}-w_{l}} ; \quad s_{u}^{0}=\mu_{0}}{w_{u}-w_{0}} .
$$

Enquanto Max $\left\{l \rho|,| f_{\mu}^{1}|,| f_{\mu}^{2} \mid\right\}>\varepsilon \quad$ Resolver

$$
\begin{aligned}
& \left(\begin{array}{ccc}
\mathbf{1} & \frac{\mathbf{1}}{2 a} & -\frac{\mathbf{1}}{2 a} \\
s_{l} & w-w_{l} & \mathbf{0} \\
-s_{u} & \mathbf{0} & w_{u}-w
\end{array}\right)\left(\begin{array}{c}
\boldsymbol{\Delta} w \\
\boldsymbol{\Delta} s_{l} \\
\boldsymbol{\Delta} s_{u}
\end{array}\right)=\left(\begin{array}{c}
\rho \\
f_{\mu}^{1} \\
f_{\mu}^{2}
\end{array}\right) \\
& \lambda=\operatorname{Min}\left\{\left|\frac{w}{\Delta w}\right|,\left|\frac{s_{l}}{\Delta s_{l}}\right|,\left|\frac{s_{u}}{\Delta s_{u}}\right|\right\} \\
& w=w+0.000 \lambda \Delta w \\
& s_{l}=s_{l}+0.999 \lambda \Delta s_{l} \\
& s_{u}=s_{u}+0.999 \lambda \Delta s_{u} \\
& \mu=\delta \mu
\end{aligned}
$$

Calcular $\quad \rho, f_{\mu,}^{1}, f_{\mu}^{2}$.

Fazer $\quad w_{m}=w$ e $y_{m}=y\left(w_{m}\right)$.

FASE 2 (Cálculo da Lâmina que Maximiza a Receita Líquida e a Lâmina "equivalente”)

$$
\begin{aligned}
\text { Fazer } \quad w & =w_{0}, \quad s_{l}=s_{l}^{0}, \quad s_{u}=s_{u}^{0} \quad \mu=\mu_{0}, \quad w_{T}=w_{m} \\
\text { Calcular } \quad \sigma & =\left(P_{c} a+s_{u}-s_{l}\right) w^{2}+w_{T}\left(P_{c} c-c_{0}\right) \\
f_{\mu}^{a} & =\mu-\left(w-w_{l}\right) s_{l} ; f_{\mu}^{4}=\mu-\left(w_{u}-w\right) s_{u} ;
\end{aligned}
$$




\section{DETERMINAÇÃO DE INTERVALOS ÓTIMOS DE IRRIGAÇÃO UTILIZANDO A BARREIRA LOGARÍTMICA}

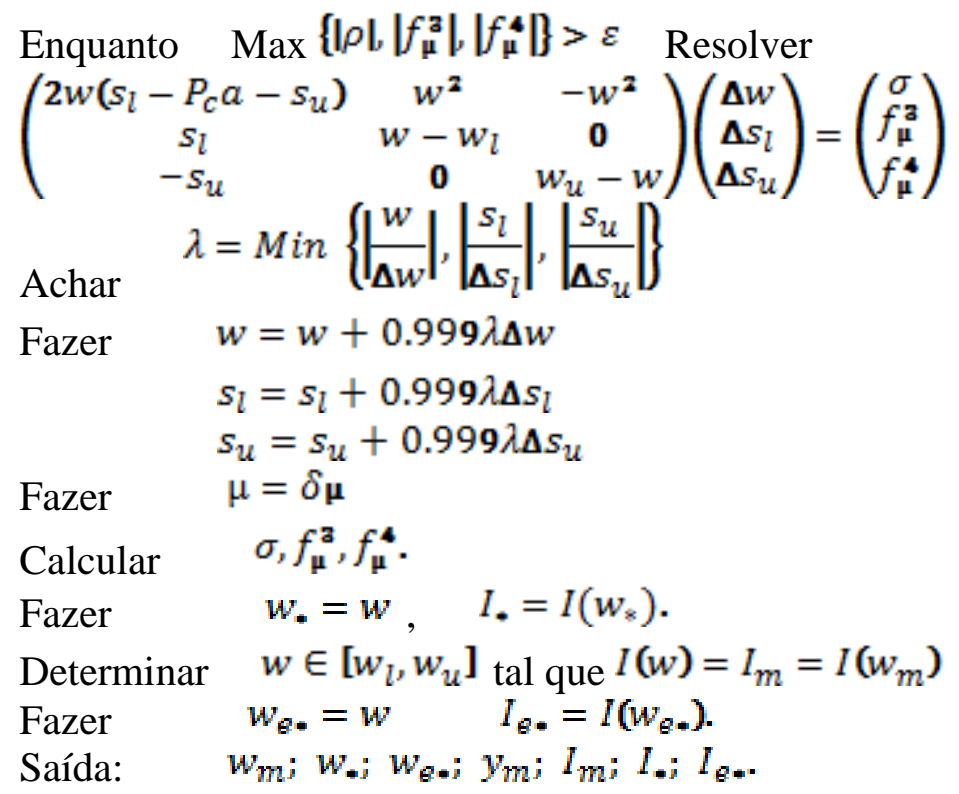

\section{REFERÊNCIAS BIBLIOGRÁFICAS}

ANDRADE JÚNIOR, A. S.; FRIZZONE, J. A.; BASTOS, E. A.; CARDOSO, M. J. e RODRIGUES, B. H. N.. Estratégias Ótimas de Irrigação para a Cultura da Melancia. Pesq. Agropec. Bras., Brasília, v. 36, n. 2, p. 301-305, fev. 2001.

DOOREMBOS, J.; KASSAM, A. H.. Efeito da Água no Rendimento das Culturas. Campina Grande, UFPB, 1994, 306 p. (FAO. Irrig. E Drenag. (33)).

ENGLISH, M. J. e RAJA, S. N..Perspectives on deficit irrigation. Agricultural Water Management, v. 32, p. 1-14. 1996.

ENGLISH, M.J. Deficit irrigation. I: Analytical Framework. Journal of the Irrigation and Drainage Engineering of ASCE, New York, v.116, n.3, p.399-412, 1990.
ENGLISH, M.J.; SOLOMON, K.H.; HOFFMAN, G.J. A paradigm shift in irrigation management. Journal of Irrigation and Drainage Engineering, New York, v.128, n.5, p.267-277, 2002.

FRIZZONE, J. A.; ANDRADE JÚNIOR, A. S.; ZOCOLER, J. L.. Planejamento da Irrigação. Análise de Decisão de Investimentos. 1 ed. Brasília. Embrapa Informação Tecnológica, 627 p.; 2005.

GONZAGA, C. C.. Path Following for Linear Programming. SIAM Review Ann Ambor, v. 34, n. 2, p. 167 - 227, 1992.

KARMARKAR, N.. A New Polynomialtime Algorithm for Linear Programming. Combinatorica, Berlin, v. 4 n. 2 p. 373 395, 1984.

YARON, D.; BRESLER, E. Economics analysis of on-farm irrigation using response functions of crops. In: HILLEL, D. (Ed.). Advances in irrigation. New York : Academic, 1983. v. 2, p. 223-255. 